\title{
Salmonella sepsis in \\ ulcerative colitis: Report of a \\ case and review of the \\ literature
}

\author{
DAVID K. YOUNG, D.O \\ Trotwood, Ohio \\ KEVIN M. REID, D.o. \\ Dayton, Ohio
}

\begin{abstract}
Coincident salmonellosis and ulcerative colitis is an uncommon and poorly understood clinical problem. The occurrence of salmonella sepsis in patients with previously diagnosed ulcerative colitis has only been reported twice in the medical literature, and only 1 case was discussed in detail. This article describes the third patient to have concurrent salmonella sepsis and ulcerative colitis - the first without gastrointestinal infection. A detailed report of the patient's long hospital course with fatal outcome is presented, analysis of the possible mechanisms of infection in the patient is discussed in depth, and the medical literature regarding the combination of ulcerative colitis and salmonella infection is reviewed. This case emphasizes the need to culture stools in any patient who presents with a presumed flare up of inflammatory bowel disease to rule out an infectious cause. Also, patients with salmonella colitis should be followed to be certain that they do not have inflammatory bowel disease as well. Antibiotic coverage is recommended for patients with known ulcerative colitis and salmonella infection, although treatment data are lacking.
\end{abstract}

Coincident ulcerative colitis and salmonella infection is an uncommon clinical occurrence. Several recent reviews ${ }^{1-4}$ of the complications of inflammatory bowel disease do not mention the problem. Salmonella sepsis in patients with inflammatory bowel disease appears to be a very rare event. Only two cases ${ }^{5,6}$ are reported to date, with both patients having sepsis develop during a course of salmonella enteritis.

We report a case of salmonella sepsis of an unknown source that developed during a severe flare up of ulcerative colitis. Possible etiologic and com- plicating factors in this case are discussed in detail, and the pertinent literature is reviewed.

\section{Report of case}

A 43-year-old white man with a 2-year history of ulcerative colitis was admitted to an outlying hospital with a complaint of bloody diarrhea of 4 days' duration. The patient had been off of sulfasalazine and prednisone therapy for 2 months and had done well since his last exacerbation 6 months prior to admission. He was placed on intravenous fluids on admission, but when his bloody stools failed to resolve, he was transferred to our institution on the eleventh hospital day for total parenteral nutrition and possible colectomy.

The patient's past medical history was remarkable only for ulcerative colitis. He did not drink alcohol and had quit smoking 18 months prior to admission. His only surgeries were three herniorrhaphies, and he was taking no medications at the time of admission. Review of systems was unremarkable.

On admission, the patient was pale and quite thin and appeared to be acutely ill. His temperature was $99.2 \mathrm{~F}$. orally, and his blood pressure was $120 / 80 \mathrm{~mm} . \mathrm{Hg}$. Abdominal examination revealed marked tenderness over the entire abdomen and tympanitic bowel sounds, but no rebound tenderness. The remainder of the physical examination was normal. Admission complete blood count revealed a hemoglobin concentration of $14.1 \mathrm{gm} . / \mathrm{dl}$., a hematocrit reading of 42.1 percent, and a leukocyte count of $14,500 /$ cu.mm., with a differential distribution of 54 percent segmented neutrophils, 8 percent band forms, 29 percent lymphocytes, and 9 percent monocytes. Other laboratory values were: erythrocyte sedimentation rate, $60 \mathrm{~mm}$./hr.; serum alkaline phosphatase, slightly elevated at $147 \mathrm{I} . \mathrm{U} . / \mathrm{L}$.; blood urea nitrogen, $25 \mathrm{mg} . / \mathrm{dl}$; serum creatinine, $1.2 \mathrm{mg}$./dl.; and serum amylase, $37 \mathrm{U} . /$ L., which is normal. A flat abdominal x-ray revealed a small bowel and right colon ileus, with no evidence of toxic megacolon. Initial stool, urine, and blood cultures did not grow pathogenic bacteria.

The patient was put at bowel rest, total parenteral nutrition was started in a central vein, and intravenous administration of $500 \mathrm{mg}$. of methylprednisolone every 6 hours was begun. He was followed closely for the development of toxic megacolon with repeated abdominal films. 
On the third hospital day, esophagogastroduodenoscopy and colonoscopy were performed. The first procedure revealed esophagitis, gastritis, and antral ulcers, and the second procedure showed clotted blood, pseudopolyposis, diffuse ulcerations, loss of haustral markings, and loss of vascularity, which was thought to be consistent with ulcerative colitis.

Bleeding continued, with fourth hospital day hemoglobin and hematocrit values of $7.5 \mathrm{gm}$./dl. and 21.1 percent, respectively, and the patient was transfused with packed red blood cells. On the sixth hospital day, a surgical consultation was obtained, but the bleeding had slowed and the recommendation was to observe the patient. Total parenteral nutrition was continued, while oral feedings were slowly resumed. The patient improved clinically and the corticosteroid dosage was gradually reduced.

On the twelfth hospital day, inflammation of both knees with effusion occurred; this was thought to be the arthritis of inflammatory bowel disease. Over the next 2 days, the abdominal pain returned along with severe cramps and bloody diarrhea, which necessitated an increase in the corticosteroid dose. Again, the patient was placed on bowel rest. A complete blood count revealed a marked left shift, with a leukocyte count of $9,700 / \mathrm{cu} . \mathrm{mm}$. and a differential count of 36 percent segmented neutrophils, 46 percent band forms, 10 percent lymphocytes, 7 percent monocytes, and 1 percent metamyelocytes. An oral temperature of $102.9 \mathrm{~F}$, peaking at $105 \mathrm{~F}$, occurred on the sixteenth hospital day. Forty-eight hours later, abdominal pain and tenderness returned, and large bowel gas without dilation was again seen on an abdominal film. The patient was placed on tobramycin $(80 \mathrm{mg}$., IV, every 8 hours), cephalothin ( 2 gm., IV, every 4 hours), clindamycin $(600 \mathrm{mg}$., IV, every 6 hours) and moxalactam (1 gm., IV, every 6 hours). This course was carried out for 2 days. Blood cultures obtained 2 days earlier revealed Salmonella enteritidis, serotype paratyphi $B$, which is sensitive to ampicillin, and the antibiotic regimen was changed to ampicillin (2 gm., IV, every 6 hours) and tobramycin ( $80 \mathrm{mg}$., IV, every 8 hours).

The patient clinically improved, with a decrease in the fever, resolution of the abdominal pain, and abatement of the bloody diarrhea. Blood cultures were rechecked 10 days after ampicillin was started, and Salmonella enteritidis, serotype paratyphi $B$ was again recovered. The subclavian catheter was changed, and computed tomography was performed to search for a possible mycotic aneurysm. The entire aorta was normal, but the spleen was enlarged, the kidneys appeared to have abscesses, and the lung fields demonstrated alveolar infiltrates bilaterally. These findings all were thought to represent septic emboli. Pulmonary consultation was obtained and the impression rendered was salmonella pneumonia due to hematogenous spread.

On the thirty-third hospital day, the patient's fever spiked to $103.3 \mathrm{~F}$, and blood cultures this time grew Candida albicans. The subclavian catheter again was changed. Three days later, the patient suffered respiratory arrest and was placed on a ventilator. Over the next 48 hours, he continued to deteriorate. Amphotericin B (5 mg., IV) was started, but the patient went into acute renal failure and septic shock and became acidotic, leukopenic, and thrombocytopenic. On the thirty-eighth hospital day, he suffered cardiac arrest and could not be resuscitated. An autopsy was refused by the family.

\section{Discussion}

\section{Literature review}

The first mention of salmonella infection in ulcerative colitis dates back to 1957 , when Banks and associates ${ }^{7}$ reported "bacillary dysentery or salmonella infections" in 8 of 245 patients with the disease. Although no details were given, these patients apparently had only gastrointestinal infection. Black and coauthors, ${ }^{8}$ in their classic review of unusual aspects of salmonellosis published in 1960, did not mention any cases of salmonella infection in inflammatory bowel disease. In 1961, $\mathrm{Hook}^{9}$ published a review of host factors that influence the development of salmonellosis in humans; 1 patient was reported to have ulcerative colitis. No details as to the type or extent of infection were given. The first detailed review of patients with salmonella infection and ulcerative colitis was presented in 1967 by Lindeman and coworkers, ${ }^{10}$ who reported 7 patients with the combination. Their cases demonstrated the difficulty in making a diagnosis on first presentation and the need for close follow up.

The occurrence of salmonella septicemia in ulcerative colitis is even less commonly reported. In 1974 , Cherubin and his group 5 discussed 412 cases of septicemia caused by nontyphoid salmonella, and only 1 patient in this group had ulcerative colitis. No clinical data on this patient are available. The most complete discussion to date was published by Dronfield, Fletcher, and Langman, ${ }^{6}$ also in 1974 . They reviewed 5 cases of coincident salmonellosis and ulcerative colitis, with all of their patients presenting with salmonella enteritis. One patient developed salmonella sepsis, and the outcome was fatal. It was the opinion of the authors that antibiotic coverage with ampicillin should be provided for patients who are receiving corticosteroid therapy for ulcerative colitis if a recent salmonella infection has been diagnosed. These cases also emphasize the need to obtain stool cultures for any patient who presents with suspected ulcerative colitis, as well as for those patients that appear to be having a flare up of known ulcerative colitis. Conversely, ulcerative colitis should be considered in any patient who has persistent bloody diarrhea after salmonella gastroenteritis, especially when bloody stools continue after the cultures have been found to be negative.

Six other cases of ulcerative colitis and salmonella infection have been reported. In 1967, in a case $^{11}$ in which salmonella infection mimicked 
acute appendicitis, the 14-year-old female patient eventually was diagnosed as having ulcerative colitis. In a 1979 report $^{12}$ on the pathology of salmonella colitis, a patient with persistent diarrhea was shown to have chronic ulcerative colitis. In 1982, Kressner and associates ${ }^{13}$ discussed a known ulcerative colitis patient who appeared to present with a flare up of inflammatory bowel disease. The patient did not respond to aggressive medical treatment, but after salmonella was cultured on the eighth hospital day, she responded to antibacterial treatment. Szilagyi and coworkers, ${ }^{14}$ in a 1985 review of salmonella infections and inflammatory bowel disease, described 3 patients with ulcerative colitis and salmonella gastroenteritis.

Salmonella traditionally has been viewed as an infection of the small bowel. Only recently has it been documented ${ }^{13,15,16}$ that salmonella can cause a colitis that is very similar in appearance to chronic ulcerative colitis. The clinical presentation, sigmoidoscopic appearance, radiographic findings, and histopathology are all indistinguishable. ${ }^{12,17,18}$ Salmonella colitis also can present as a clinical picture resembling Crohn's disease. ${ }^{17,19}$ Toxic megacolon has even been reported ${ }^{17,20}$ to occur with salmonella colitis.

\section{Comments on present case}

The remainder of the discussion will focus on the possible etiologies and complicating factors involving our patient's infection. The first possibility is that the presumed flare up of ulcerative colitis was instead an acute salmonella colitis. This is unlikely for two reasons. One is that on admission, several cultures of both stool and blood did not recover salmonella, and several aspirates obtained on the third hospital day during the sigmoidoscopic procedure did not grow pathogenic organisms. Also, repeat stool cultures obtained during the second exacerbation of bloody diarrhea did not grow salmonella. The other reason for salmonella colitis being unlikely was the initial excellent response of the flare up to corticosteroids.

A second consideration is that salmonella may have been transmitted during endoscopy, as had been reported in several articles. ${ }^{21-25}$ This has been known to be responsible for in-hospital epidemics. ${ }^{23}$ Patients have been reported to have salmonella in stool, blood, urine, and sputum, and in a peritoneal abscess-all transmitted from an endoscope. Again, this source was considered doubtful for our patient because the stool cultures did not reveal salmonella, and all cases reported to date have had salmonella in the stool. The endoscopes used were not specifically cultured for salmonella, and they had already been cleaned and reused for several days. In addition, no other reports of positive salmonella cultures were received from our microbiology laboratory. Also, there were no in-hospital infections reported during the period of time the patient was in the hospital.

A very interesting situation, which has only recently been described, is overwhelming infection in ulcerative colitis resulting from hyposplenism. Ryan and associates ${ }^{26}$ in 1978 published a report on the splenic function of patients with inflammatory bowel disease. Their studies revealed hyposplenism in 13 of 36 patients with ulcerative colitis. Hyposplenism was seen only in patients with extensive colonic involvement who either were having a relapse or an active, persistent course of disease. The 13 patients had Howell-Jolly bodies in a peripheral smear, a prolonged clearance of heat-damaged, $\mathrm{Cr}$ labeled red blood cells, and small spleens. Four of these patients had septic complications after surgery, and the authors' conclusion was that the hyposplenism contributed to the sepsis. Foster and coauthors $^{27}$ in 1982 described a case in which a patient with long-standing ulcerative colitis developed overwhelming pneumococcal sepsis and died. On necropsy, the patient's spleen was noted to be markedly atropic, and it was concluded that the patient had hyposplenism due to ulcerative colitis. The patient apparently had been in clinical remission at the time of the septic episode, unlike the patients in Ryan and associates'26 report. Our patient did not develop Howell-Jolly bodies, and his spleen was, in fact, enlarged on the computed tomographic scan. Therefore, this explanation is not likely. The link between hyposplenism and ulcerative colitis is fascinating and deserves further study.

Another possibility in our case is that one of the previous flare ups of ulcerative colitis was actually an episode of salmonella enteritis, and that the patient was harboring the organism in his biliary tract. Again, however, this scenario is doubtful, because salmonella was not recovered from the stool.

Food contamination is the most common mode of transmission for salmonella. This is an unlikely source in our patient, again because the stools were sterile. Also, no other cultures were reported positive for salmonella in other patients, and no food-borne epidemic occurred in our hospital during this time.

The final point to be discussed is the contribution of glucocorticoids. The risk of infection with corticosteroid use has been well documented. ${ }^{28}$ Corticosteroids have many effects on both humoral and cellular mechanisms, but it appears that effects on the cellular aspects of immune function are most important. ${ }^{29}$ In view of the frequency of 
corticosteroids being used in treatment of ulcerative colitis, it is surprising that more cases of sepsis and infection in these patients are not reported. In Kirsner's ${ }^{2}$ article on complications in inflammatory bowel disease, infection was mentioned, but without detailed discussion. ${ }^{2}$ The acquired immunodeficiency syndrome (AIDS) is characterized by a profound decrease in cellular immunity, and a 1986 paper by Fischl and coauthors $^{30}$ reports salmonella bacteremia in 6 patients with AIDS. These patients were similar to the patient we present in that none of the 6 had a foci of infection discovered and 5 had relapses of bacteremia. The authors postulated an inability in AIDS patients to eradicate salmonella. The decrease in cellular immunity observed with highdose corticosteroid use resembles that seen in AIDS. Similar mechanisms may be involved in ulcerative colitis patients taking corticosteroids and in AIDS patients with regard to difficulty in eliminating salmonella. The combination of steroid use and the recently described hyposplenism would seem to place these patients at high risk for infection.

\section{Conclusion}

We report the case of a patient with severe ulcerative colitis who died from complications of salmonella sepsis. We believe that this is the first report of an ulcerative colitis patient who developed salmonella bacteremia without gastrointestinal infection. A literature review revealed 20 previously described patients with salmonella infection and ulcerative colitis (Table 1). Several possible sources of our patient's infection were discussed and eliminated, and the etiology remains unknown.

Salmonella bacteremia appears to be a rare complication of ulcerative colitis, but one that can be fatal. Also, as several cases in the literature have demonstrated, it can be very difficult to distinguish ulcerative colitis from salmonella colitis, and initial treatment may be jeopardized if an incorrect diagnosis is made. Any patient with a flare up of ulcerative colitis should have the stools cultured to rule out enteric infection, and, conversely, patients with salmonella enteritis should be followed carefully to be certain that ulcerative colitis does not coexist. Dronfield, Fletcher, and Langman ${ }^{6}$ believe that when some uncertainty exists, steroid use, if

\begin{tabular}{|c|c|c|c|c|}
\hline Author(s) & $\begin{array}{c}\text { No. of } \\
\text { patients }\end{array}$ & $\begin{array}{l}\text { Blood cultures } \\
\text { for salmonella }\end{array}$ & $\begin{array}{l}\text { Stool cultures } \\
\text { for salmonella }\end{array}$ & Outcome \\
\hline $\begin{array}{l}\text { Cherubin } \\
\text { and associates }\end{array}$ & 1 & $(+)$ & NR & Unknown \\
\hline $\begin{array}{l}\text { Dronfield, } \\
\text { Fletcher, and }\end{array}$ & & & & \\
\hline Langman $^{6}$ & 5 & $\begin{array}{c}(+) \\
1 \text { patient }\end{array}$ & $\begin{array}{l}(+) \\
\text { all } 5 \text { patients }\end{array}$ & $\begin{array}{l}1 \text { died, } \\
\text { salmonella } \\
\text { sepsis }\end{array}$ \\
\hline Hook $^{9}$ & 1 & $\mathrm{NR}^{*}$ & $(+) \dagger$ & Unknown \\
\hline $\begin{array}{l}\text { Lindemann } \\
\text { and associates }\end{array}$ & 7 & NR & $\begin{array}{c}(+) \\
\text { all } 7 \text { patients }\end{array}$ & $\begin{array}{l}1 \text { died, cause } \\
\text { unknown }\end{array}$ \\
\hline $\begin{array}{l}\text { Swartz } \\
\quad \text { and associates }\end{array}$ & 1 & NR & $(+)$ & Survived \\
\hline $\begin{array}{l}\text { McGovern and } \\
\text { Slavutin }^{12}\end{array}$ & 1 & NR & $(+)$ & Survived \\
\hline $\begin{array}{l}\text { Kressner } \\
\quad \text { and associates }{ }^{13}\end{array}$ & 1 & $(-) \ddagger$ & $(+)$ & Survived \\
\hline $\begin{array}{l}\text { Szalagyi and } \\
\text { associates }^{14}\end{array}$ & 3 & NR & $(+)$ & \\
\hline $\begin{array}{r}\text { Young and Reid } \\
\text { (present case) }\end{array}$ & 1 & $(+)$ & $\begin{array}{l}\text { all } 3 \text { patients } \\
\qquad \underline{(-)}\end{array}$ & $\begin{array}{c}\text { Improved } \\
\text { Died, } \\
\text { salmonella } \\
\text { sepsis }\end{array}$ \\
\hline Total & 21 & 3 & 19 & $\begin{array}{l}2 \text { died of } \\
\text { salmonella } \\
\text { sepsis }\end{array}$ \\
\hline $\begin{array}{l}{ }^{*} \mathrm{NR}=\text { not reported } \\
\dagger(+)=\text { culture positi } \\
\ddagger(-)=\text { culture negati }\end{array}$ & & & & \\
\hline
\end{tabular}


any, should be combined with an antibiotic regimen. This procedure is, of course, unproved, but the advice seems sound.

1. Greenstein, A.J., Janowitz, H.D., and Sachar, D.B.: The extra-intestinal complications of Crohn's disease and ulcerative colitis. A study of 700 patients. Medicine 55:401-12, Sep 76

2. Kirsner, J.B.: The local and systemic complications of inflammatory bowel disease. JAMA 242:1177-83, 14 Sep 79

3. Kirsner, J.B., and Shorter, R.G.: Recent developments in nonspecific inflammatory bowel disease. N Engl J Med 306:(Pt. 1) 775-85, 1 Apr 82; and 306:(Pt. 2) 837-48, 8 Apr 82

4. Janowitz, H.D., and Sachar, D.B.: Inflammatory bowel disease. Adv Intern Med 27:205-46, 1982

5. Cherubin, C.E., et al.: Septicemia with non-typhoid salmonella. Medicine 53:365-76, Sep 74

6. Dronfield, M.W., Fletcher, J., and Langman, M.J.S.: Coincident salmonella infections and ulcerative colitis. Problems of recognition and management. Br Med J 1:99-100, 19 Jun 74

7. Banks, B.M., Korelitz, B.I., and Zetzel, L.: The course of nonspecific ulcerative colitis. Review of twenty years' experience and late results. Gastroenterology 32:983-1012, Jun 57

8. Black, P.H., Kunz, L.J., and Swartz, M.N.: Salmonellosis. A review of some unusual aspects. N Engl J Med 262:(Pt. 1) 811-7, 21 Apr 60; 262:(Pt. 2) 864-70, 28 Apr 60; and 262:(Pt. 3) 921-7, 5 May 60

9. Hook, E.W.: Salmonellosis. Certain factors influencing the interaction of salmonella and the human host. Bull NY Acad Med 37:499-512, Jul 61 10. Lindeman, R.J., et al.: Ulcerative colitis and intestinal salmonellosis. Am J Med Sci 254:855-61, Dec 67

11. Swartz, M.N., et al.: Salmonella infections. Clin Pediatr 6:33-40, Jan 67

12. McGovern, V.J., and Slavutin, L.J.: Pathology of salmonella colitis. Am J Surg Pathol 3:483-90, Dec 79

13. Kressner, M.S., et al.: Salmonellosis complicating ulcerative colitis. Treatment with trimethoprim-sulfamethoxazole. JAMA 248:584-5, 6 Aug 82

14. Szilagyi, A., et al.: Salmonella infections complicating inflammatory bowel disease. J Clin Gastroenterol 7:251-5, June 85

15. Mandal, B.K., and Mani, V.: Colonic involvement in salmonellosis. Lancet $1: 887-8,24$ Apr 76

16. Saffouri, B., Bartolomeo, R.S., and Fuchs, B.: Colonic involvement in salmonellosis. Dig Dis Sci 24:203-8, Mar 79

17. Pennington, C.R., Bickerstaff, K.I., and Lyall, M.H.: Salmonella in- fection with colitis. J Infect 2:181-3, 1980

18. Murphy, T.F., and Gorbach, S.L.: Salmonella colitis. NY State J Med $82: 1236-8$, Jul 82

19. Vender, R.J., and Marignani, P.: Salmonella colitis presenting as a segmental colitis resembling Crohn's disease. Dig Dis Sci 28:848-51, Sep 83

20. Schofield, P.F., Mandal, B.K., and Ironside, A.G.: Toxic dilatation of the colon in salmonella colitis and inflammatory bowel disease. Br J Surg 66:5-8, Jan 79

21. Beecham, H.J., III, Cohen, M.L., and Parkin, W.E.: Salmonella typhimurium. Transmission by fiberoptic upper gastrointestinal endoscopy. JAMA 241:1013-5, 9 Mar 79

22. O'Connor, B.H., et al.: Salmonellosis infection transmitted by fiberoptic endoscopes. Lancet $2: 864-6,16$ Oct 82

23. Chmel, H., and Armstrong, D.: Salmonella oslo. A focal outbreak in a hospital. Am J Med 60:203-8, Feb 76

24. Dean, A.G.: Transmission of Salmonella typhi by fiberoptic endoscopy (letter). Lancet 2:134, 16 Jul 77

25. Editorial: Fibreoptic infections. Lancet 2:782-3, 11 Oct 80

26. Ryan, F.P., et al.: Hyposplenism in inflammatory bowel disease. Gut 19:50-5, Jan 78

27. Foster, K.J., et al.: Overwhelming pneumococcal septicaemia in a patient with ulcerative colitis and splenic atrophy. Gut 23:630-2, Jul 82 28. Fauci, A.S., Dale, D.C., and Balow, J.E.: Glucocorticosteroid therapy. Mechanisms of action and clinical considerations. Ann Intern Med $84: 304-15$, Mar 76

29. Grieco, M.H.: The role of corticosteroid therapy in infection. Hosp Pract 19:131-43, Mar 84

30. Fischl, M.A., et al.: Salmonella bacteremia as manifestation of acquired immunodeficiency syndrome. Arch Intern Med 146:113-5, Jan 86

Accepted for publication in October 1986. Updating, as necessary, has been done by the authors.

At the time this paper was written, Dr. Young was a resident in internal medicine at Grandview Hospital, Dayton, Ohio. Dr. Larry Klein was director of residency training at that time. Dr. Young is now a pulmonary fellow at Grandview. Dr. Reid is in private practice of internal medicine in Dayton, Ohio, and is on the staff of Grandview Hospital.

Dr. Young, 420 Outer Belle Road, Trotwood, Ohio 45426. 